\title{
Variations of Neck Structures after Supracricoid Partial Laryngectomy: A Multislice Computed Tomography Evaluation
}

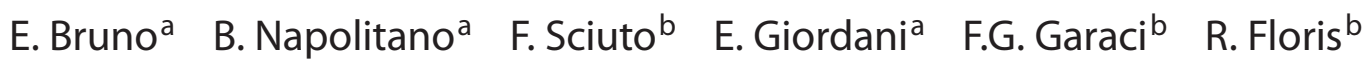 \\ M. Alessandrini ${ }^{a}$ S. Di Girolamo ${ }^{a} \quad$ F. Ottaviani ${ }^{a}$
}

Departments of a Otolaryngology and biagnostic Imaging and Interventional Radiology, Tor Vergata University, Roma, Italy

\section{Key Words}

Larynx $\cdot$ Partial laryngectomy $\cdot$ Cricohyoidoepiglottopexy Multislice computed tomography

\begin{abstract}
Background: Surgery of laryngeal cancer used to profoundly alter the anatomy of the cervical region. Accurate anatomo-embryologic studies and repeated surgical trials allowed recognition of the cricoarytenoid complex as the smallest anatomofunctional unit able to maintain all the laryngeal functions. Objectives: The aim of this study was to determine whether significant variations of neck anatomical parameters exist after partial laryngectomy, and to analyze whether some of these parameters are associated with a positive functional outcome. Methods: Out of 48 patients treated with a surgical technique according to Mayer-Piquet (cricohyoidoepiglottopexy, CHEP) over a 6-year period, 18 patients were enrolled in the study. Patients were all males with a mean age of 60 years. Cervical structures and their relationships were measured by computed tomography, and the measurements before and after surgery were compared. Results: Our data showed that hyoid bone is modified, both in morphology and position during CHEP. More specifically the relation of the hyoid bone to other neck structures (identified by the $\alpha$-angle) is modified. The neolarynx and trachea undergo a caudocranial shift. All diame-
\end{abstract}

ters of the cricoid cartilage remain unchanged after surgery. The position of the epiglottis after CHEP, in particular its relation with the arytenoid cartilage, is closely related to swallowing function outcome and recovery time. Conclusions: Our study showed that these structures, and more specifically the relations among them, undergo significant variations after CHEP. Our results identify some parameters, i.e. the $\alpha$-angle, width of the hyoid bone and position of the epiglottis, that may predispose to a positive functional outcome after surgery.

Copyright $\odot 2007$ S. Karger AG, Basel

\section{Introduction}

Surgery of laryngeal cancer used to profoundly alter the anatomy of the cervical region. Throughout the years, increasing attention has been given to preserve both the anatomical and functional integrity of the larynx while achieving a high local control.

Accurate anatomo-embryologic studies and repeated surgical trials allowed recognition of the cricoarytenoid complex as the 'smallest anatomofunctional unit' able to maintain all the laryngeal functions: breathing, swallowing and phonation [1]. It is a common experience of most surgeons that patients undergoing partial laryngectomy with cricohyoidoepiglottopexy (CHEP) or cricohyoido-

\section{KARGER \\ Fax +41613061234 E-Mail karger@karger.ch} www.karger.com (c) 2007 S. Karger AG, Basel

0301-1569/07/0695-0265\$23.50/0

Accessible online at:

www.karger.com/orl
Ernesto Bruno

Policlinico Tor Vergata, Department of Otolaryngology

Viale Oxford, 81

IT-00133 Roma (Italy)

Tel. +39 0620902 925, Fax+39 0620902 921, E-Mail digirolamo@med.uniroma2.it 
Table 1. Anatomical parameters of anterior cervical structures in controls and patients after CHEP, expressed as mean of the single values

\begin{tabular}{|c|c|c|c|c|c|c|c|c|c|}
\hline & $\mathrm{AI},{ }^{\circ}$ & La.I.Max, mm & La.I.Min, mm & $\mathrm{HI}, \mathrm{mm}$ & $\alpha,{ }^{\circ}$ & $\mathrm{OAP}, \mathrm{mm}$ & IAP, mm & $\mathrm{OL}, \mathrm{mm}$ & IL, $\mathrm{mm}$ \\
\hline Controls & 29.5 & 42.0 & 18.7 & 36.1 & 17.8 & 25.0 & 18.0 & 30.8 & 18.0 \\
\hline Patients after CHEP & 32.7 & 44.2 & 18.0 & 35.5 & 32.5 & 28.4 & 16.9 & 30.3 & 18.4 \\
\hline
\end{tabular}

$\mathrm{AI}=$ Hyoid angle defined as the angle identified by a line perpendicular to the main axis and passing through the midpoint of the body of the hyoid bone and by another line joining the midpoint of the body of the hyoid bone and the distal end of the greater cornua of the hyoid bone; La.I.Max = the distance between the two greater cornua of the hyoid bone; La.I.Min = width of the body of the hyoid bone; $\mathrm{HI}=$ anteroposterior length of the hyoid bone; $\alpha$ = virtual line joining the midpoint of the lower margin of the body of the mandible and the upper face of the third cervical vertebra and another line joining the midpoint of the lower margin of the body of the mandible and the midpoint of the body of the hyoid bone; OAP = the outer anteroposterior diameter of the cricoid cartilage; IAP = inner anteroposterior diameter of the cricoid cartilage; $\mathrm{OL}=$ outer lateral diameter of the cricoid cartilage; IL = inner lateral diameter of the cricoid cartilage.

pexy may show varying degrees of functional recovery [2-4]. They may range from an eventful recovery within 7 days to a clinical pattern of dysphagia and dyspnea, requiring nasogastric feeding tube and tracheostomy tube for several months [1].

Recent generation of computed tomography (CT) scanners (multislice CT) [5-7], thanks to a rapid and detailed images acquisition, seem to be excellent tools for evaluating the varying degrees of morphological modifications occurring after CHEP. Initially and throughout the years, morphological studies of the larynx as well as of other anatomical structures used to be performed postmortem on fixed preparations. Subsequently, the introduction of CT scanners made possible to add to the morphological study a morphometric study of the larynx on fixed cadaveric structures as well as on patients [8] and, more recently, to perform a functional imaging of the larynx [9] and small airways [10]. However, as CT and other imaging techniques have been more extensively employed in clinical practice, the importance of developing an evaluation technique by defining the planes and points of measurements has been recognized as a critical landmark able to yield reproducible images $[11,12]$.

The aim of this study was to identify the existence of anatomical parameters, whose variations after CHEP may be associated with a positive functional outcome. Furthermore, we wished to evaluate if some of these variations may be predicted preoperatively in order to identify preoperative parameters that predispose to positive functional outcome. This evaluation might be useful to predict preoperatively the surgical outcome guiding the best intraoperative choices according to the specific anatomy of the single patient.
We have focused on detecting the anatomical changes of anterior cervical structures following partial surgery of the larynx by multislice CT. Such a technology is able to analyze the variation of the parameters in the followup of the operated patient.

\section{Subjects and Methods}

Out of 48 patients treated with a surgical technique according to Mayer-Piquet over a 6-year period (1998-2004), 18 patients were enrolled in the study. Patients were all males with a mean age of 60 years (range: $54-68$ years). Data were also obtained in a population of subjects ( 30 subjects, all males, mean age: 55 years) undergoing a CT of the larynx for diagnostic purposes other than a preoperative evaluation, and these subject were considered as controls (table 1).

The selected patients underwent both morphologic and clinical evaluation.

\section{Clinical Evaluation}

The functional outcome after surgery was clinically evaluated with regard to time of permanence of the tracheostomy tube inserted (to evaluate breathing), time of nasogastric feeding tube inserted (to evaluate swallowing), duration of hospitalization and incidence of complications.

\section{Morphologic Evaluation}

The morphologic evaluation consisted of multislice CT (General Electric Milwaukee, USA) study of neck structures without endovenous contrast medium and fiberoptic laryngoscopy, before and after surgery. Morphological changes, as measured by $\mathrm{CT}$ and endoscopy, were then compared. In order to obtain standardized measurements during the CT study, the subject was positioned with his neck in a 'neutral' posture (not flexed nor hyperextended), so that the virtual axis joining the midpoint of the lower margin of the body of the jaw and the upper face of the third cervical vertebra was perpendicular to the line passing through 


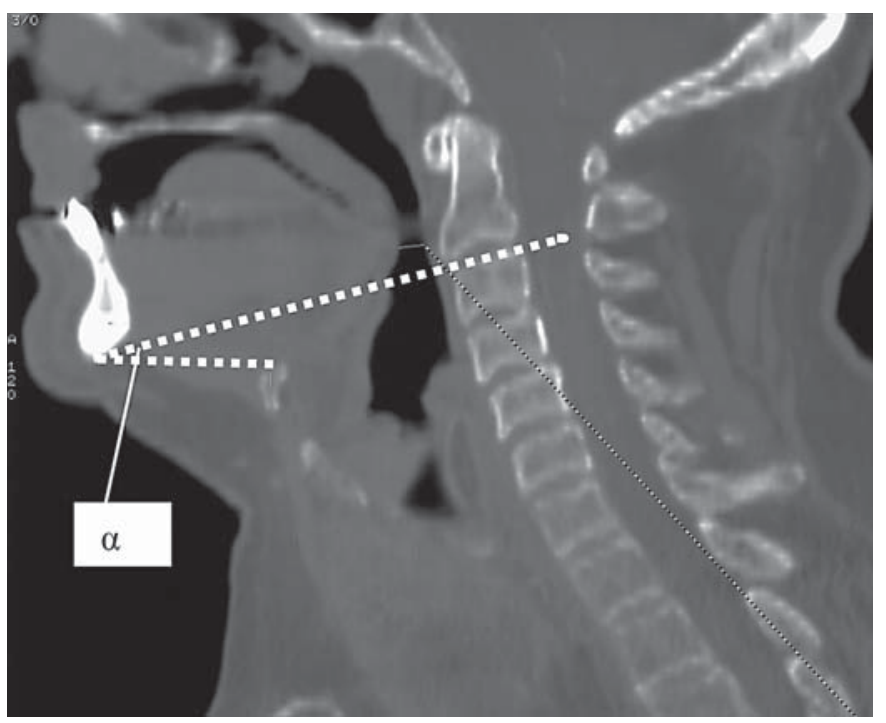

Fig. 1. $\alpha$-Angle measured in a patient before surgery. The measure was calculated on MPR sagittal plane from multislice CT without contrast media.

the posterior margins of cervical vertebrae, with an error equal to $\pm 1^{\circ}$.

\section{CT Acquisition}

CT exam started with the acquisition of two guide images (scanogram), to evaluate the correct position of the neck. Images were acquired without administration of endovenous contrast medium, with volumetric technique (thickness: $0.6 \mathrm{~mm}$; gap: $3 \mathrm{~mm} / \mathrm{s}$ ). Once axial images were obtained, some selected variables were measured by means of an electronic ruler in the selected patients, enabling a detailed comparison before and after surgery. It should be emphasized that the morphometric analysis was performed on routinary, basal CT acquisitions, though it did not require additional X-ray exposure, pain or discomfort for the patient and further costs and time for the radiologist. The radiological examination performed for this study was not more invasive than any other CT scan commonly used as a diagnostic tool for laryngeal carcinoma.

\section{Variables}

Measurements were performed in $\mathrm{mm}$ for lengths, degrees for angles and $\mathrm{mm}^{2}$ for surfaces.

Structures considered were in particular the hyoid bone, the arytenoid cartilage, the cricoid cartilage and the trachea; the $\alpha$-angle was defined. The $\alpha$-angle was identified by a virtual line joining the midpoint of the lower margin of the body of the mandible and the upper face of the third cervical vertebra and another line joining the midpoint of the lower margin of the body of the mandible and the midpoint of the body of the hyoid bone (fig. 1,2). This angle was recognized to define the relation of hyoid bone to other neck structures.

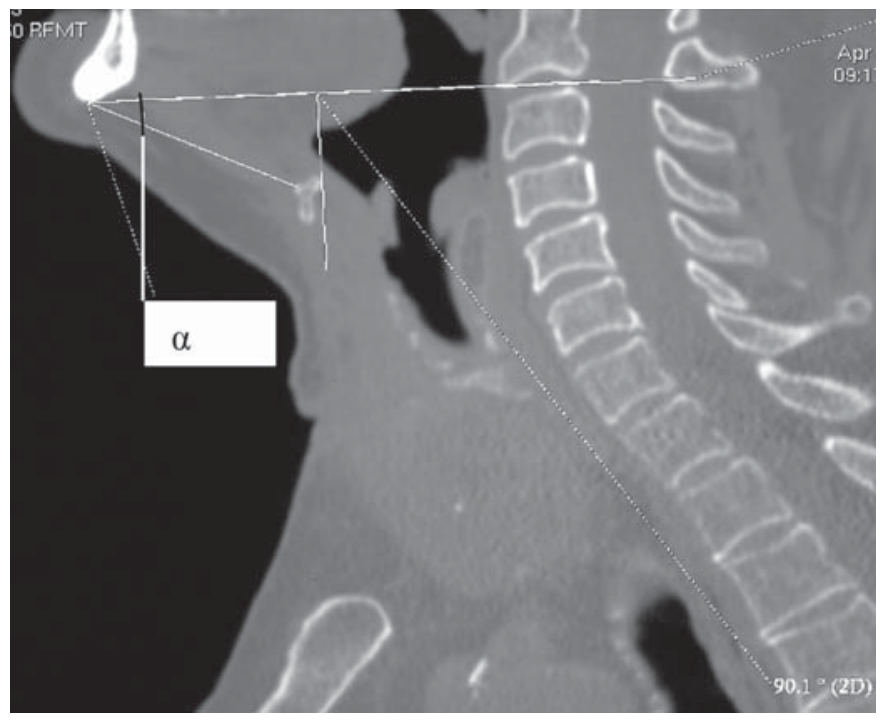

Fig. 2. $\alpha$-Angle measured in a patient after surgery. The measure was calculated on MPR sagittal plane from multislice CT without contrast media.

In order to evaluate the hyoid bone, we considered the following variables: (a) the distance between the greater cornua; (b) width of the body; (c) anteroposterior length; (d) hyoid angle, defined as the angle identified by a line perpendicular to the main axis and passing through the midpoint of the body of the hyoid bone and by another line joining the midpoint of the body of the hyoid bone and the distal end of the greater cornua of the hyoid bone (fig. 3). For the cricoid cartilage, we considered: (a) the outer anteroposterior (OAP) diameter; (b) inner anteroposterior (IAP) diameter; (c) outer lateral (OL) diameter; (d) inner lateral (IL) diameter (fig. 4).

A statistical analysis of variations of the measured variables before and after surgery was performed using the Student's t test for paired data (SPSS for Windows) with a p value of 0.05 or less defined as significant. The variations of the measured variables after surgery as compared to control subjects were analyzed using the Student's $t$ test for unpaired data (SPSS for Windows) with a $\mathrm{p}$ value of 0.05 or less defined as significant.

\section{Results}

\section{Clinical Results}

The decannulation rate was $100 \%$. The mean time until decannulation was 8 days (range: 6-12 days). The mean time for removal of the nasogastric feeding tube was 11 days (range: 4-17 days).

Normal swallowing was achieved in $94.4 \%$ of patients $(17 / 18)$ in the 1st postoperative month. Pneumonia from 


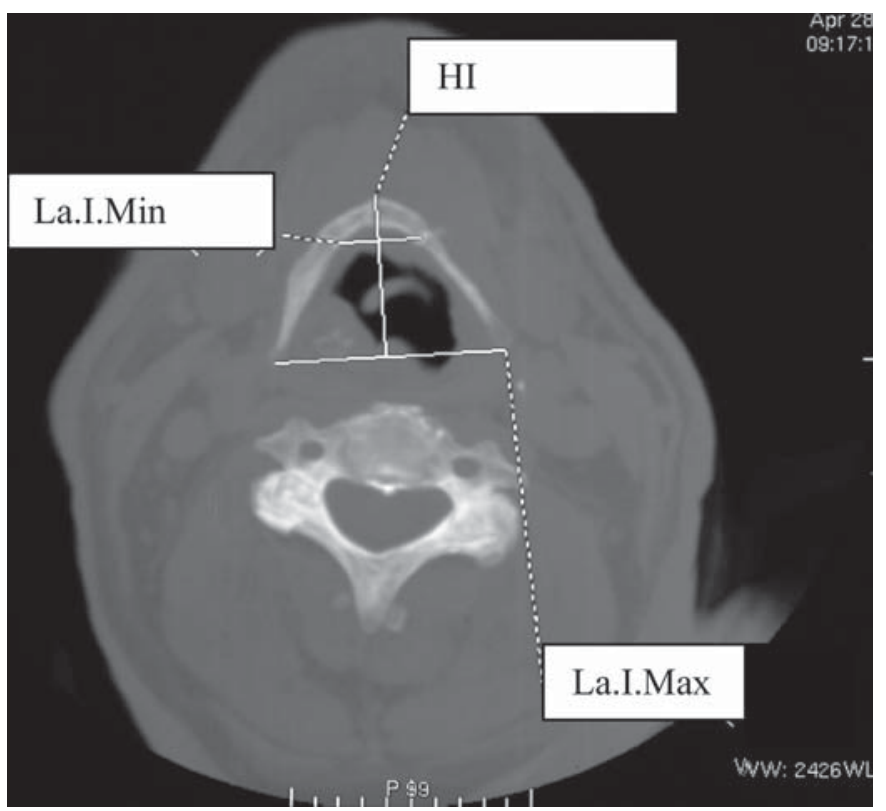

Fig. 3. Hyoid bone measures. The measure was calculated on MPR axial plane from multislice CT without contrast media.

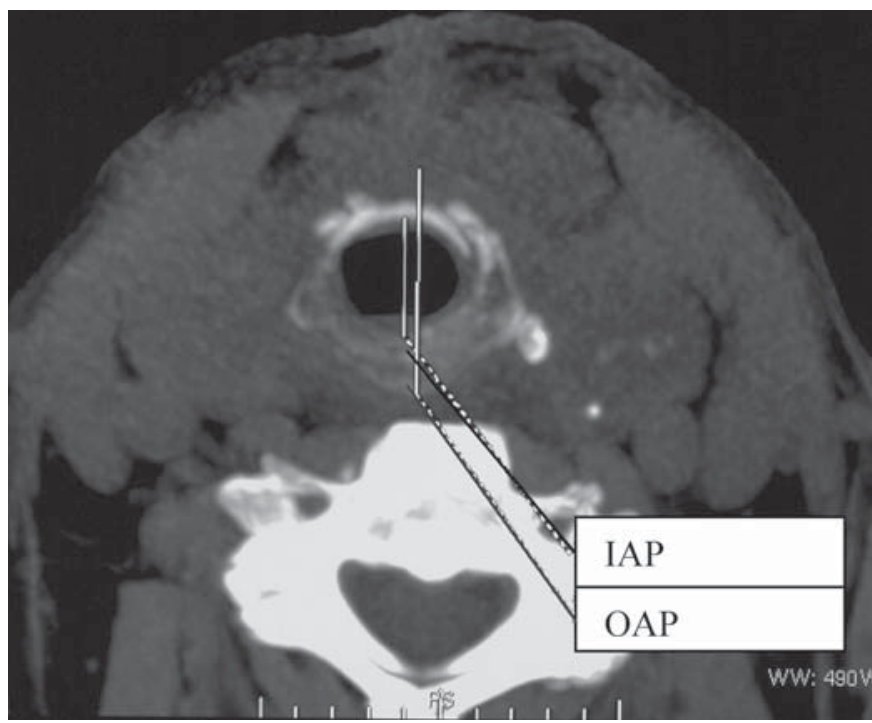

Fig. 4. Cricoid cartilage measures. The measure was calculated on MPR axial plane from multislice CT without contrast media.

aspiration occurred in $11 \%$ of patients (2/18): it was successfully managed with antibiotics, but required longer hospitalization and repositioning of the nasogastric feeding tube.

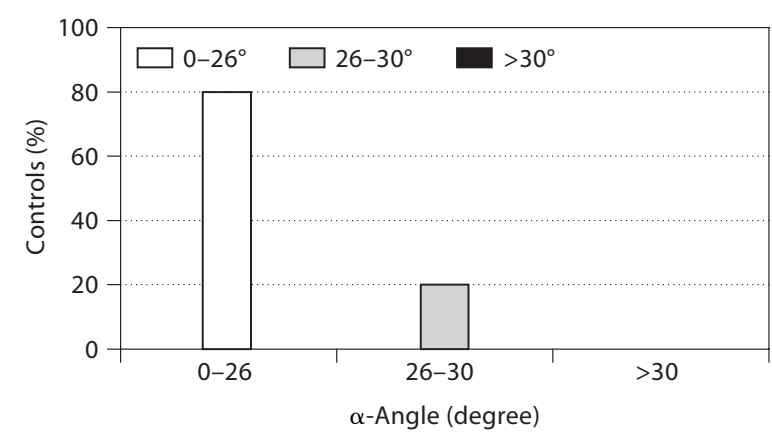

Fig. 5. $\alpha$-Angle in controls.

The mean duration of hospitalization was 19 days (1330 days).

\section{Morphologic Results}

Our data show that the hyoid bone is modified both in morphology and position during CHEP. In particular, the distance between the greater cornua increases in $33.3 \%$ of patients $(6 / 18)$, decreases in $44.4 \%$ of patients $(8 / 18)$, and remains unchanged in $22.2 \%$ of patients $(4 / 18)$. Relating these data to the clinical history of the patient, we observed that those patients (44\%) with a reduced distance between the greater cornua of the hyoid bone showed a better recovery of the swallowing function when compared to patients with increased distance. In fact, this distance might affect the size of the hypopharyngeal tube and its mobility.

The $\alpha$-angle $(\mathrm{p}<0.01)$ increased significantly in amplitude: before surgery it showed a mean value of $17.7^{\circ}$ (range: $3.6-28.6^{\circ}$ ), after surgery a mean value of $32.5^{\circ}$ (range: $27.1-35.3^{\circ}$ ). $\alpha$-Angle values have then been compared between CHEP patients before surgery and a population of normal subjects. The range of $\alpha$-angle values in normal subjects $\left(0-30^{\circ}\right)$ and CHEP patients before surgery $\left(3.6-28.6^{\circ}\right)$ differed only for the larger size of normal subject series. It has been observed that $80 \%$ of normal subjects showed an $\alpha$-angle ranging between 0 and $27^{\circ}$ (fig. 5), whereas none of the CHEP patients had an $\alpha$ angle $<27^{\circ}$ (fig. 6). Furthermore, $78 \%$ of these patients presented an angle over $30^{\circ}$. Twenty percent of both controls and patients had the $\alpha$-angle ranging between 27 and $30^{\circ}$. Patients presenting with a high-amplitude $\alpha$-angle $\left(>34.5^{\circ}\right)$ showed a prompt and complete recovery of all laryngeal functions. Conversely, patients with a low $\alpha$-angle $\left(<30.1^{\circ}\right)$ showed a protracted and complicated 


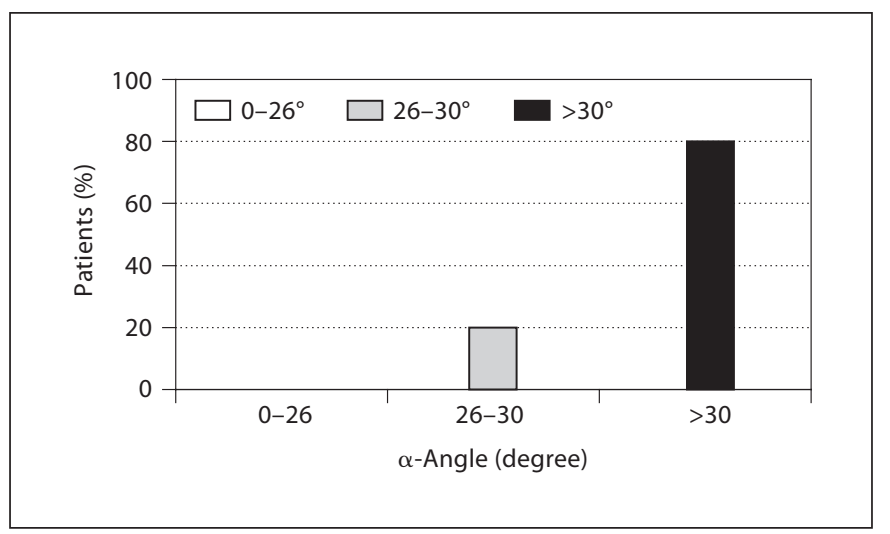

Fig. 6. $\alpha$-Angle in patients after CHEP.

recovery. Moreover, we observed that low preoperative $\alpha$-angle values $\left(<7^{\circ}\right)$ predispose to lower postoperative $\alpha$-angle values $\left(<30.1^{\circ}\right)$ due to the specific anatomical relations among cervical structures (fig. 1, 2). Therefore, patients with low preoperative $\alpha$-angle values showed poor postoperative functional recovery on the basis of hospitalization time (23-30 days in patients with preoperative $\alpha$-angle values $<7^{\circ}$ vs. $13-20$ days in patients with preoperative $\alpha$-angle values $>7^{\circ}$ ) and time to achieve a normal swallowing function (11-17 days in patients with preoperative $\alpha$-angle values $<7^{\circ}$ vs. $4-9$ days in patients with preoperative $\alpha$-angle values $>7^{\circ}$ ).

The neolarynx and trachea undergo a caudocranial shift. This is confirmed by the position of the cricoid cartilage that is localized at the level of the 7th cervical vertebra before surgery and moves to the level of the 5th cervical vertebra after CHEP (in some cases, also between the 4th and 5th vertebrae). It is interesting to observe that the cranial displacement of the trachea and neolarynx, due to a reduced length of the laryngeal organ for resection of the thyroid cartilage, is not affected by the preoperative length of the thyroid cartilage whichever it was (mean: $42.9 \mathrm{~mm}$, range: $35.9-52.5 \mathrm{~mm}$ ).

We observed that all diameters (OAP, IAP, OL, IL) of the cricoid cartilage remain unchanged after surgery. This may explain the good recovery of respiratory function in all patients (decannulation rate: 100\%): none of the patients showed any respiratory complication, and those with a larger cricoid diameter showed a better respiratory recovery.

We observed that the position of the epiglottis after CHEP, in particular its relation to other neck structures, is highly related to swallowing function outcome and recovery time. Patients (11\%) in whom epiglottis was posi- tioned during CHEP below the residual arytenoid cartilage showed swallowing problems: 2 patients started to swallow after the 15th postsurgical day and developed ab ingestis pneumonia. Conversely, patients (89\%) in whom epiglottis was correctly positioned during CHEP above the residual arytenoid cartilage showed a complete recovery of the swallowing function within 12 days.

The obtained images were then compared with the ones obtained with fiberoptic laryngoscopy, showing high sensitivity (96\%) and specificity (99\%).

\section{Discussion}

Partial laryngectomy (CHEP) represents the most appropriate surgical choice in glottic carcinoma with impaired motility of the vocal cord, with no involvement of the arytenoid cartilage [13]. CHEP results in the resection of the entire thyroid cartilage, both vocal cords and one arytenoid cartilage (from the tumor-bearing side) with preservation of its posterior mucosa.

The neolarynx is subsequently formed by the cricoid cartilage and a portion of the epiglottis cartilage both anchored to the hyoid bone and one residual arytenoid: the reduced height of neolarynx results in a cranial displacement of the neoglottic space. This surgical procedure allows avoiding, in many cases, total laryngectomy, with the epiglottis and the residual arytenoid preserving all the laryngeal functions (breathing, swallowing and phonatory function) [1].

The anatomical study of anterior cervical structures by means of multislice CT showed that these structures, and more specifically the relations among them, undergo significant variations after CHEP. It has been noted that these variations may affect the surgical functional outcome and some of them may be determined by the preoperative anatomy.

The thyrohyoid membrane is removed during surgery and the hyoid bone is anchored directly to the cricoid cartilage, without any other interposed structure. Therefore, the greater traction applied by an osteocartilagineous structure rather than a membranous one might favor the inferior displacement of the hyoid bone. We measured this displacement as an increased amplitude of the $\alpha$-angle, which is present in all operated patients. However, patients with high $\alpha$-angle values showed a complete recovery of all laryngeal functions, probably due to a better reorganization of the relationships among the base of the tongue, neopyriform sinuses and epiglottis. Conversely, patients with a low $\alpha$-angle showed a protracted 
recovery. In addition, it was noted that low preoperative $\alpha$-angle values predispose to lower postoperative $\alpha$-angle values due to the specific anatomical relations among cervical structures, and patients with low preoperative $\alpha$-angle values show poor postoperative functional recovery. The greater traction exerted by the neolarynx on the hyoid bone causes a modification of the anatomy of this bone, which is related to recovery of swallowing function. It seems that the preoperative anatomy of cervical structures may determine, as a response to the cranial displacement of neolarynx, either an enlargement or a narrowing of the hyoid bone and therefore a poorer or better swallowing function.

It has been noted that the position of the epiglottis after CHEP is closely related to swallowing function outcome and recovery time. In particular, patients show either a complete recovery of swallowing function or swallowing complications if the epiglottis is positioned either above or below the residual arytenoid cartilage. Multislice CT appeared to be extremely helpful in evaluating preoperatively both the position and size of epiglottis cartilage in order to plan the best intra-operative choice for a correct placement of the cartilage.

Our results identify some parameters, such as the $\alpha$ angle, width of the hyoid bone, position of the epiglottis and its relation to the arytenoid cartilage, which undergo significant variations after CHEP and whose variations are associated with a different functional outcome. Some of these variations ( $\alpha$-angle, position of epiglottis) may be determined preoperatively. They are able to predict the surgical functional outcome. Therefore, they may help presurgical planning and guide the best intraoperative choices according to the specific anatomical relations among the anterior cervical structures.

We would like to emphasize that multislice CT, because of its ability to provide higher resolution images as compared to spiral CT images, plays an important role in the definition of the above-mentioned anatomical relations.

\section{Conclusion}

We believe that a preoperative CT study of each patient undergoing CHEP should consider the $\alpha$-angle, width of the hyoid bone and position of the epiglottis as critical radiological landmarks able to predict the postoperative functional outcome.

Therefore, we would like to emphasize that a close collaboration between the otolaryngologist and the radiologist in the evaluation of patient candidates for a partial laryngectomy would be helpful in presurgical planning, intraoperative guidance, and postoperative analysis of the treated anatomic regions, assuring a better recovery from a surgical procedure, anyway successful as oncologic outcome.

\section{References}

1 Naudo P, Laccourreye O, Weinstein G, et al: Complications and functional outcome after supracricoid partial laryngectomy with cricohyoidoepiglottopexy. Otolaryngol Head Neck Surg 1998;118:124-129.

2 Bussi M, Riontino E, Cardarelli L, et al: Cricohyoidoepiglottopexy: deglution in 44 cases. Acta Otorhinolaryngol Ital 2000;20:442447.

3 Crevier-Buchman L, Laccourreye O, Wuyts FL, et al: Comparison and evolution of perceptual and acoustic characteristic of voice after supracricoid partial laryngectomy with cricohyoidoepiglottopexy. Acta Otolaryngol 1998;118:594-599.

4 Brusori S, Soro A, Tesei F, et al: Deglutition in patients treated with reconstructive laryngectomy. Radiol Med (Torino) 1998;95: $154-160$.
5 Vannier MW: Evaluation of 3D imaging. Crit Rev Diagn Imaging 2000;41:315-378.

6 Pavone P, Panebianco V, Laghi A, et al: Virtual endoscopy with a volumetric recostruction technique: the technical aspects. Radiol Med (Torino) 1998;95:618-623.

7 Satava RM: Virtual Endoscopy. Surg Endosc 1996;10:173-174.

8 Hudgins PA, Siegel J, Jacobs I, et al: The normal pediatric larynx on CT and MR. Am J Neuroradiol 1997;18:239-245.

9 Lell MM, Gress H, Hothorn T, et al: Multiplanar functional imaging of the larynx and hypopharynx with multislice spiral CT. Eur Radiol 2004;14:2198-2205.
10 Tanaka N, Matsumoto T, Suda H, et al: Paired inspiratory-expiratory thin-section CT finding in patients with small airway disease. Eur Radiol 2001;11:393-401.

11 Friedrich G, Kainz J: Morphometry of the larynx in horizontal sections. Normal data for the quantitative evaluation of current imaging technics. Laryngol Rhinol Otol (Stuttg) 1988;67:269-274.

12 Hermans R, Feron M, Bellon E, et al: Laryngeal tumor volume measurements determined with CT: a study on intra-and interobserver variability. Int J Radiat Oncol Biol Phys 1998;40:553-557.

13 Laccourreye O, Muscatello L, Laccourreye L, et al: Supracricoid partial laryngectomy with cricohyoidoepiglottopexy for 'early' glottic carcinoma classified as T1-T2N0 invading the anterior commissure. Am J Otolaryngol 1997;18:385-390 